\title{
Comprehensive insights into transcriptional adaptation of intracellular mycobacteria by microbe-enriched dual RNA sequencing
}

Rienk A Rienksma', Maria Suarez-Diez ${ }^{1}$, Hans-Joachim Mollenkopf², Gregory M Dolganov³, Anca Dorhoi', Gary K Schoolnik ${ }^{3}$, Vitor AP Martins dos Santos ${ }^{1,5}$, Stefan HE Kaufmann ${ }^{4}$, Peter J Schaap ${ }^{1 *}$ and Martin Gengenbacher ${ }^{4,6}$

\begin{abstract}
Background: The human pathogen Mycobacterium tuberculosis has the capacity to escape eradication by professional phagocytes. During infection, $M$. tuberculosis resists the harsh environment of phagosomes and actively manipulates macrophages and dendritic cells to ensure prolonged intracellular survival. In contrast to other intracellular pathogens, it has remained difficult to capture the transcriptome of mycobacteria during infection due to an unfavorable host-to-pathogen ratio.
\end{abstract}

Results: We infected the human macrophage-like cell line THP-1 with the attenuated $M$. tuberculosis surrogate M. bovis Bacillus Calmette-Guérin (M. bovis BCG). Mycobacterial RNA was up to 1000-fold underrepresented in total RNA preparations of infected host cells. We employed microbial enrichment combined with specific ribosomal RNA depletion to simultaneously analyze the transcriptional responses of host and pathogen during infection by dual RNA sequencing. Our results confirm that mycobacterial pathways for cholesterol degradation and iron acquisition are upregulated during infection. In addition, genes involved in the methylcitrate cycle, aspartate metabolism and recycling of mycolic acids were induced. In response to $M$. bovis BCG infection, host cells upregulated de novo cholesterol biosynthesis presumably to compensate for the loss of this metabolite by bacterial catabolism.

Conclusions: Dual RNA sequencing allows simultaneous capture of the global transcriptome of host and pathogen, during infection. However, mycobacteria remained problematic due to their relatively low number per host cell resulting in an unfavorable bacterium-to-host RNA ratio. Here, we use a strategy that combines enrichment for bacterial transcripts and dual RNA sequencing to provide the most comprehensive transcriptome of intracellular mycobacteria to date. The knowledge acquired into the pathogen and host pathways regulated during infection may contribute to a solid basis for the deployment of novel intervention strategies to tackle infection.

Keywords: Mycobacterium bovis BCG, THP-1 cells, Infection, Host-microbe interaction, Transcriptome, Dual RNA sequencing, Microbe enrichment

\section{Background}

Tuberculosis (TB) is an infectious disease caused by the airborne pathogen Mycobacterium tuberculosis and accounts for 1.3 million fatalities annually [1]. Unlike nonpathogenic microbes that are eliminated inside the maturing phagosome of immune cells such as macrophages, $M$. tuberculosis brings phagosome maturation to

\footnotetext{
* Correspondence: peter.schaap@wur.nl

'Laboratory of Systems and Synthetic Biology, Wageningen University and Research Centre, Dreijenplein 10,6703, HB, Wageningen, the Netherlands Full list of author information is available at the end of the article
}

a halt and manages to cope with various host threats including acidification, reactive radicals and nutrient limitation [2]. Studying the transcriptome of intracellular pathogens, in particular M. tuberculosis, during infection remained difficult due to a low bacteria-to-host RNA ratio. For different pathogens the number of organisms per host cell spans several orders of magnitudes ranging from 1 to 10 for $M$. tuberculosis and up to 1000 for Chlamydia [3,4].

The first insights into the intracellular life of $M$. tuberculosis provided by comparative microarray analysis, 
revealed a switch from aerobic to anaerobic respiration, induction of the dormancy regulon $\operatorname{dos} R$ and iron scavenging as well as upregulation of $\beta$-oxidation of fatty acids upon infection [5]. Similar technologies and quantitative real-time PCR were applied to broaden our understanding of specific aspects of intracellular $M$. tuberculosis [6-9]. Microarray probes have the disadvantage of unspecific cross-hybridization between pathogen and host [4], and most often such probes are not optimized for minimal cross-reactivity with other species. Cappelli and colleagues [8] estimated that non-specific signals account for up to $12.5 \%$ of all signals. Additionally, transcription of non-coding regions and missed or miss-annotated genes often remain disregarded due to a limited array design. Quantitative real-time PCR has mostly been applied to small subsets of genes, since detection of each transcript requires a pair of specific oligonucleotides [6-9].

Dual RNA sequencing (dual RNA-seq) is a relatively novel technique to study gene expression profiles. This technique allows unbiased and simultaneous sequencing of transcriptomes of multiple organisms and therefore is a superb technology to study intracellular pathogens during infection of host cells. The sequencing reads can subsequently be matched in-silico to the respective organism. Without prior knowledge of sample content, its composition can be deduced from dual RNA-seq datasets without targeting specific species [10]. Most importantly, dual RNA-seq captures the transcriptome in its entirety thereby overcoming the limitations of microarrays discussed above. First application of this technology to study $M$. avium subsp. paratuberculosis during macrophage infection has shed new light on mycobacterial iron acquisition [11].

The attenuated TB vaccine strain $M$. bovis Bacillus Calmette-Guérin ( $M$. bovis BCG) has been widely used in research as surrogate for pathogenic $M$. tuberculosis due to a high degree of genome identity [12-14]. In this study, we investigated the transcriptional adaptation of $M$. bovis BCG 24 hours after infection of the human macrophagelike cell line THP-1 by dual RNA-seq. The underrepresentation of bacterial RNA in preparations of total RNA from infected host cells requires high sequencing depth to gain statistical significance and adequate pathogen coverage, leading to increased costs. Mangan and colleagues developed a method entailing differential lysis with guanidine thiocyanate to enrich for mycobacteria from infected macrophages, thus avoiding massive underrepresentation of bacterial RNA as compared to total RNA preparations of infected cells [15]. This method has been used for in vivo transcriptome studies using microarrays $[6,16]$. Here we present a strategy that combines bacterial enrichment for bacterial transcripts and dual RNA-seq, which we evaluate against non-enriched samples.

\section{Results}

Twenty-four hours post-infection, THP-1 cells were harvested and total RNA was isolated. Additionally, two out of three infected THP-1 samples were enriched for $M$. bovis BCG bacilli, using the procedure described in Methods. The analysis of the 50-bp RNA-derived paired-end sequencing data is illustrated in Figure 1. Two out of the three datasets derived from the nonenriched infections (IF1/2) were compared to a reference sample with uninfected THP-1 cells (THP) and differentially expressed THP-1 genes were identified. For differential $M$. bovis BCG gene and small RNA expression analysis, the datasets derived from enriched infections (IF1/2ER) were compared to a reference culture of exponentially growing $M$. bovis BCG (EGB). A spike-in sample (SPI) was used to estimate the percentage of infected cells and to correlate the reads of spiked-in M. bovis BCG with the $M$. bovis BCG culture and the nonenriched THP-1 infections with M. bovis BCG. An overview of the primary sequencing data is depicted in Table 1.

\section{Pathogen specific enrichment strategy is effective}

It has been estimated that a minimum of $2-5$ million reads from a ribosomal RNA-depleted library is required to adequately cover the gene expression profile of a pathogen in a dual RNA-seq experiment [17-19]. Datasets IF0, IF1 and IF2, derived from non-enriched infections contained $0.4,1.6$ and 1.1 million 50 -bp reads that aligned to the $M$. bovis BCG genome, which was too low for significant coverage of the gene expression profile. Subsequently, an enrichment strategy for $M$. bovis BCG was applied to overcome this obstacle, thereby increasing the coverage of intracellular $M$. bovis BCG transcripts. These enriched datasets (IF1ER and IF2ER) contained 6.1 and 3.3 million 50-bp reads that aligned to the M. bovis BCG genome (Table 1).

The absolute number of $M$. bovis BCG reads of all infected sample preparations was subsequently classified in four different categories: protein-coding RNA, ribosomal RNA, small RNA, and other (Figure 2A). We simulated the relationship between the number of identified differentially expressed protein-coding $M$. bovis BCG genes and sequencing depth (Figure 2B). For very low numbers of sequencing reads, the number of identified genes increases in a linear way with the library size. With increasing library size the number of correct identifications tends to stabilize (Figure 2B). The relative abundance of the four different categories was fairly similar in both, enriched and non-enriched samples, demonstrating that impact of enrichment per se on M. bovis BCG derived sequencing reads is negligible (Figure 2C). The normalized counts of the protein coding $M$. bovis BCG transcripts in the enriched datasets (IF1ER and 


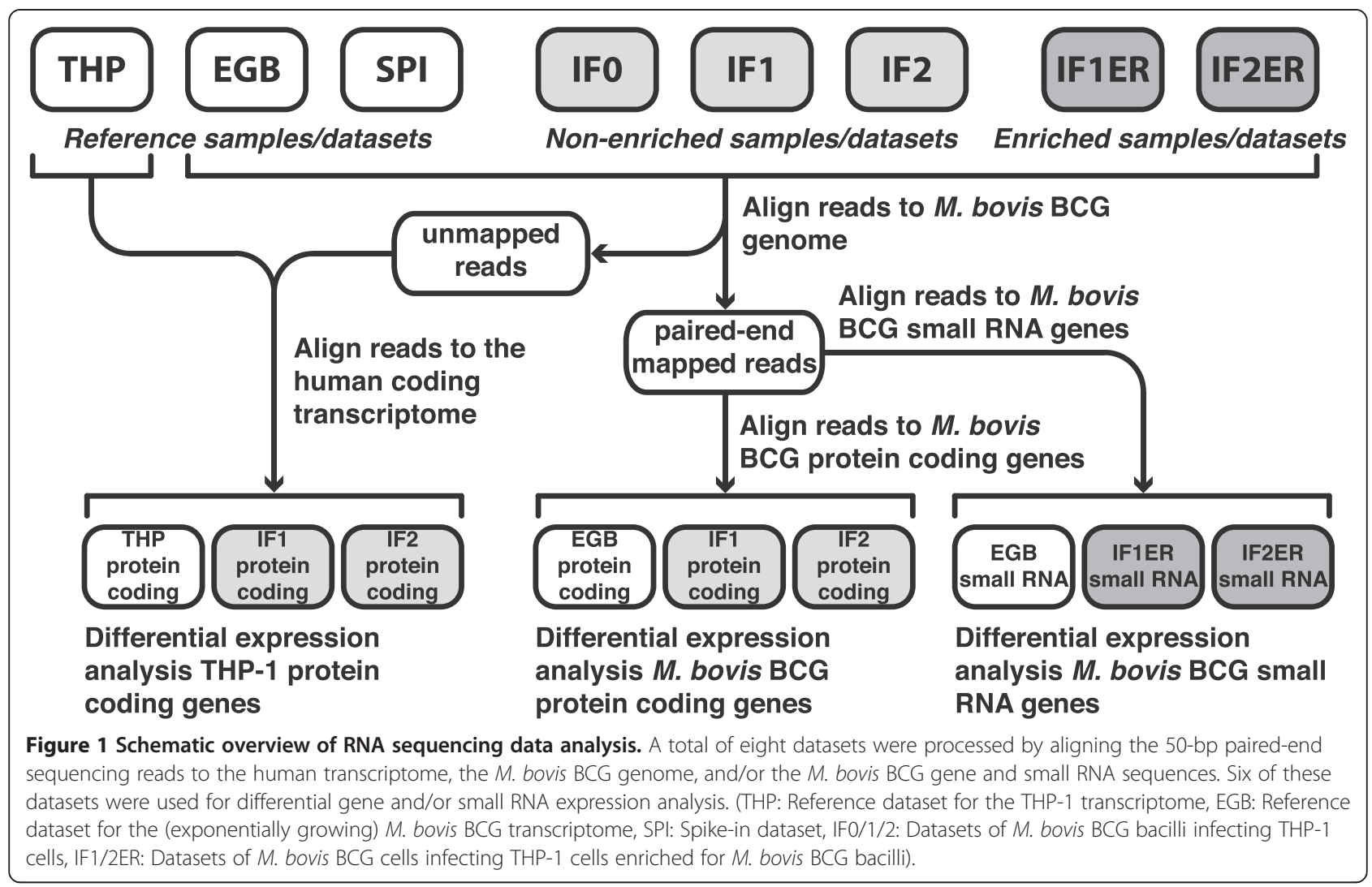

IF2ER) and the non-enriched datasets (IF1 and IF2) revealed a linear relationship, with Pearson's correlation coefficients of 0.91 and 0.92, respectively (Additional file 1). We conclude that pathogen enrichment does not introduce any bias to protein-coding gene expression of $M$. bovis BCG. However, the correlation for normalized counts per gene of THP-1 reads between the same datasets is much lower, 0.57 and 0.70, respectively (Additional file 1). Therefore, the non-enriched datasets (IF1 and IF2) were used for differential gene expression analysis of THP-1 genes. This enrichment procedure thus enabled us to study the intracellular gene expression of $M$. bovis BCG during infection.
M. bovis BCG response to infection

Twenty-four hours post-infection a clear response of the phagocytosed $M$. bovis BCG bacilli can be observed on the transcriptome level. A total of $367 M$. bovis BCG genes were differentially expressed (FDR $<0.05$ ), of which 216 were induced and 151 were repressed. A list of all differentially expressed genes of both $M$. bovis BCG and THP-1 cells is provided in Additional file 2.

\section{M. bovis BCG cholesterol catabolism genes are induced} during infection

Cholesterol is a complex lipid that consists of three cyclohexane rings (A, B and C), a cyclopentane ring (D), and an

Table 1 Reads (millions and percentages) mapped on the human transcriptome and the $M$. bovis BCG genome

\begin{tabular}{|c|c|c|c|c|c|c|}
\hline \multirow[b]{2}{*}{ Dataset } & \multirow[b]{2}{*}{ Description } & \multicolumn{2}{|c|}{ Human transcriptome } & \multicolumn{2}{|c|}{ M. bovis BCG genome } & \multirow[b]{2}{*}{ Total } \\
\hline & & $\mathrm{M}$ of reads & $\%$ & $\mathrm{M}$ of reads & $\%$ & \\
\hline THP & Uninfected THP-1 cells & 30.9 & 100 & - & - & 30.9 \\
\hline $\mathrm{EGB}$ & M. bovis BCG bacilli & - & - & 168 & 100 & 168 \\
\hline SPI & Mixed THP-1 and M. bovis BCG RNA & 31.8 & 91.0 & 3.16 & 9.0 & 35.0 \\
\hline IF0 & Infected THP-1 cells replicate 0 & 21.5 & 98.0 & 0.45 & 2.0 & 21.9 \\
\hline IF1 & Infected THP-1 cells replicate 1 & 38.2 & 96.0 & 1.57 & 4.0 & 39.7 \\
\hline IF2 & Infected THP-1 cells replicate 2 & 28.0 & 96.3 & 1.07 & 3.7 & 29.0 \\
\hline IF1ER & Infected THP-1 cells replicate 1 enriched for $M$. bovis BCG bacilli & 18.0 & 74.7 & 6.09 & 25.3 & 24.0 \\
\hline IF2ER & Infected THP-1 cells replicate 2 enriched for $M$. bovis BCG bacilli & 26.0 & 88.6 & 3.35 & 11.4 & 29.4 \\
\hline
\end{tabular}



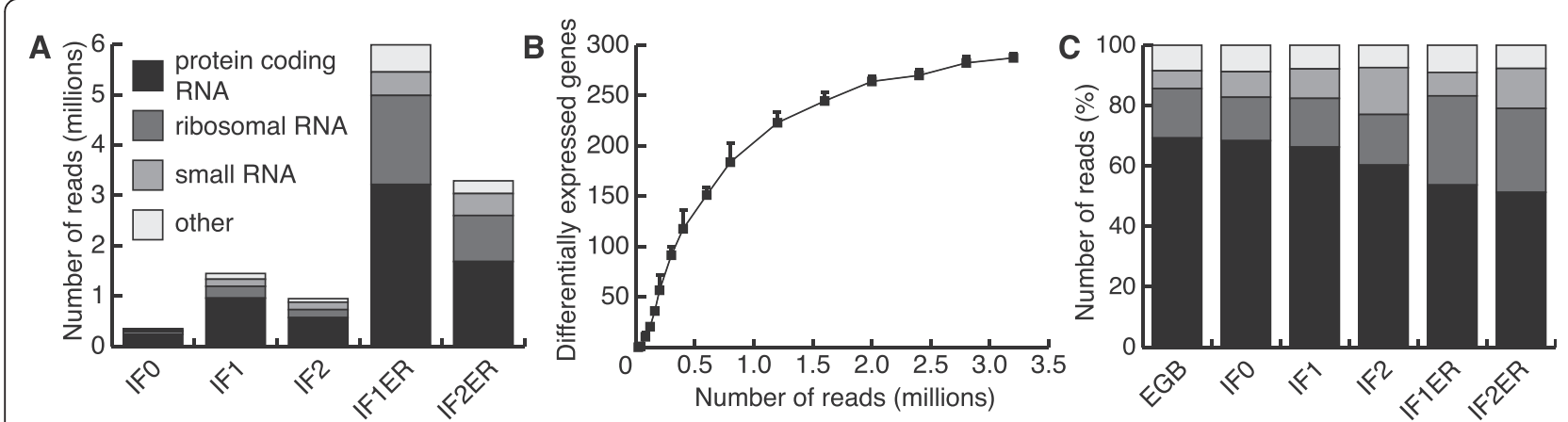

Figure $\mathbf{2}$ Classification of $\mathbf{5 0 - b p}$ sequencing reads and effect of increasing sequencing depth. (A) The total number of 50-bp sequencing reads, matching the paired-end analysis criterion that both reads could be aligned to the $M$. bovis BCG genome, were assigned to four different categories (protein-coding RNA, ribosomal RNA, small RNA, and other). The total of the reads for each sample represents the number of reads aligning to the $M$. bovis BCG genome. (B) Simulation of the relation between the number of differentially expressed $M$. bovis BCG genes and sequencing depth. Random subsets of reads were selected from EGB, IF1ER and IF2ER and the mean number ( $n=5$ ) of reliably identified differentially expressed genes (FDR < 0.05) and the standard deviation (error bars) are given for various sequencing depths. Note that the ratio of a random set to the total set approaches 1 as the size of the random set increases. Therefore, the random samples become more similar to each other and the standard deviation decreases. For reasons of completeness, we have included a standard deviation for every point. (C) Classification of the relative number of 50-bp paired-end sequencing reads aligning to the $M$. bovis BCG genome. The legend is the same as in (A).

8-carbon side chain. An incomplete degradation pathway of cholesterol was recently proposed for M. tuberculosis [20]. This pathway was extended with the side chain degradation of rings $C$ and $D$ (Additional file 3) and several genes involved in the pathway were added based on additional literature [21-28]. This extended cholesterol degradation pathway has been previously described in a genome-scale metabolic model of $M$. tuberculosis [29].

We observed a strong increase in expression of almost all genes assigned to cholesterol degradation (Figure 3A and Additional file 3). Initially, cholesterol is taken up by the transport system encoded by the mce4 gene cluster [30]. The 3 $\beta$-hydroxyl group is oxidized and isomerized to cholest-4-en-3-one either by the membrane-bound oxidase ChoD or by the dehydrogenase HsdD [21,31]. No apparent induction of the mce4 operon, the hydroxysteroid dehydrogenase (HsdD) and cholesterol oxidase (ChoD) coding genes was observed in our datasets. However, the number of transcript reads assigned to the mce4 operon and to $c h o D$ and $h s d D$ indicate that they were expressed in both the infectious and the noninfectious state (Data set S1).

Although the degradation of rings $\mathrm{A}$ and $\mathrm{B}$ is well established, the side chain degradation of rings $C$ and $D$ (Figure 3A and Additional file 3) is less understood in mycobacteria and therefore was reconstructed based on orthology with Rhodococcus equi genes [22,26].

KstR and KstR2 (BCG3639, BCG3621c; Rv3574, Rv3557c) have been previously identified as regulators of cholesterol utilization in mycobacteria [32]. The KstR2 regulon comprises $k s t R 2$ itself and all genes linked to the degradation of the side chain of rings $C$ and $D$, whereas genes regulated by KstR participate in the degradation of rings $\mathrm{A}$ and
$B$ and the initial degradation of the cholesterol side-chain (Figure 3A and Additional file 3). In our datasets the expression of $k s t R 2$ was strongly induced upon infection, whereas $k s t R$ remained unchanged (Data set S1). To verify these findings and the expression of other genes we selected a subset of 14 genes, of which 3 encode small RNAs, and designed primers (Additional file 4) to use for qRT-PCR. Among the selected genes, 5 genes are involved in cholesterol catabolism and 2 genes encode enzymes of the methylcitrate cycle (Additional file 5). The qRT-PCR results confirmed the integrity of our RNA-seq data.

We analyzed the behavior of the genes in the cholesterol degradation pathway in a compendium of expression data collected for $M$. tuberculosis. Although no condition associated with cholesterol utilization has been included in the compendium, many conditions in our compendium lead to differential expression of genes regulated by KstR and KstR2 (Figure 4). Yet, a reduced set of KstR2-regulated genes ( $f a d D 3, f a d E 31$ and ipdA) exists, which seems to be specifically induced upon infection and most likely specifically reacts to only this kind of perturbation. The specific induction renders fadD3, fadE31 and ipdA of potential interest for therapeutic intervention. Bioinformatics analysis using the consensus IdeR binding motif [33] and the KstR2 binding motif [32] revealed that these regions overlap (Figure 5).

Griffin and co-workers [34] found that although propionyl-CoA can be derived from other host metabolites, the requirement for the methylcitrate cycle is largely attributable to the degradation of host cholesterol. The induction of the methylcitrate cycle and the slight repression of icd1 (BCG3409c; Rv3339c), encoding an isocitrate dehydrogenase, suggests that the oxidative part of the citric 



Figure 3 Metabolic processes during infection. Genes in green are induced upon infection (FDR $<0.05)$, genes in red are repressed (FDR $<0.05$ ) and genes in black show no differential expression. (A) Cholesterol degradation is divided in three parts: The degradation of the side chain (yellow part), degradation of rings $A$ and $B$ (red part) and the degradation of the side chain of rings $C$ and $D$ (blue part). Dashed arrows represent multiple reactions. The degradation of the rings $C$ and $D$ side chain is based on homologous genes from Rhodococcus equi. AD: 4-androstenedione, ADD: 1,4androstenedione, 9OHADD: 9-hydroxy-1,4, androstene-3-17-dione, 3-HSA: 3-hydroxy-9,10-seconandrost-1,3,5(10)-triene-9,17-dione 3,4-DHSA: 3,4-dihydroxy-9,10-seconandrost-1,3,5(10)-triene-9,17-dione 4,9 DSHA: 4,5-9,10-diseco-3-hydroxy-5,9,17-trioxoandrosta-1 (10),2-diene-4-oic acid, HIP: 9,17-dioxo-1,2,3,4,10,19-hexanorandrostan-5-oic acid, 5OH-HIP: 5-hydroxy-methylhexahydro-1-indanone propionate. (B) Aspartate could be imported via AnsP2 and used for the synthesis of vitamin B5, glutamate and methionine. thrB, dapA and nadABC are downregulated, indicating that aspartate is to a lesser extent used to synthesize threonine, lysine and NAD(P).

acid cycle is bypassed in favor of this pathway (Additional file 6). This emphasizes that cholesterol is the main carbon source for intra-phagosomal M. bovis BCG.

\section{Expression profile suggests $M$. bovis BCG recycles mycolic acids}

Mycobacterial fatty acids are precursors for mycolic acids and are synthesized by at least two fatty acid synthases: FAS-I and FAS-II [35]. FAS-I consists of a single multifunctional enzyme, encoded by fas (BCG2545c; Rv2524c), and elongates fatty acids at the beginning of the mycolic acid synthesis pathway, while FAS-II consists of multiple enzymes and elongates fatty acids created by FAS-I. The mycobacterial genes umaA1, cmaA2, hadA, and mmaA3 (BCG0509, BCG0546c, BCG0684, BCG0692c; Rv0469, Rv0503c, Rv0635, Rv0643) encode enzymes that further process FAS-II products (Figure 6). Previous reports suggested that FadE23 and FadE24 (BCG3163, BCG3162; Rv3140, Rv3139) might be involved in recycling of mycolic acids [24]. Taken together, the expression patterns observed in our study (Figure 6) indicate that new acids are rather generated by remodeling existing mycolic acids and host fatty acids than synthesized de-novo.

\section{Expression pattern of intracellular M. bovis BCG suggests utilization of host aspartate}

Gouzy and colleagues showed that nitrogen incorporation from exogenous aspartate is required for host colonization by $M$. tuberculosis [36]. We observed changes in the gene expression pattern upon infection, regarding aspartate metabolism (Figure 3B). Intriguingly, the gene encoding the unique aspartate importer AnsP1 (BCG2144; Rv2127) showed no significant change in expression, while its homolog ansP2 (BCG0385c; Rv0346c) showed a two-fold induction (Data set S1). Gouzy and colleagues found that an M. tuberculosis ansP2-knock-out (KO) mutant was able to grow on aspartate as sole nitrogen source [36]. Moreover, an ansP1 mutant showed no growth defect in either 


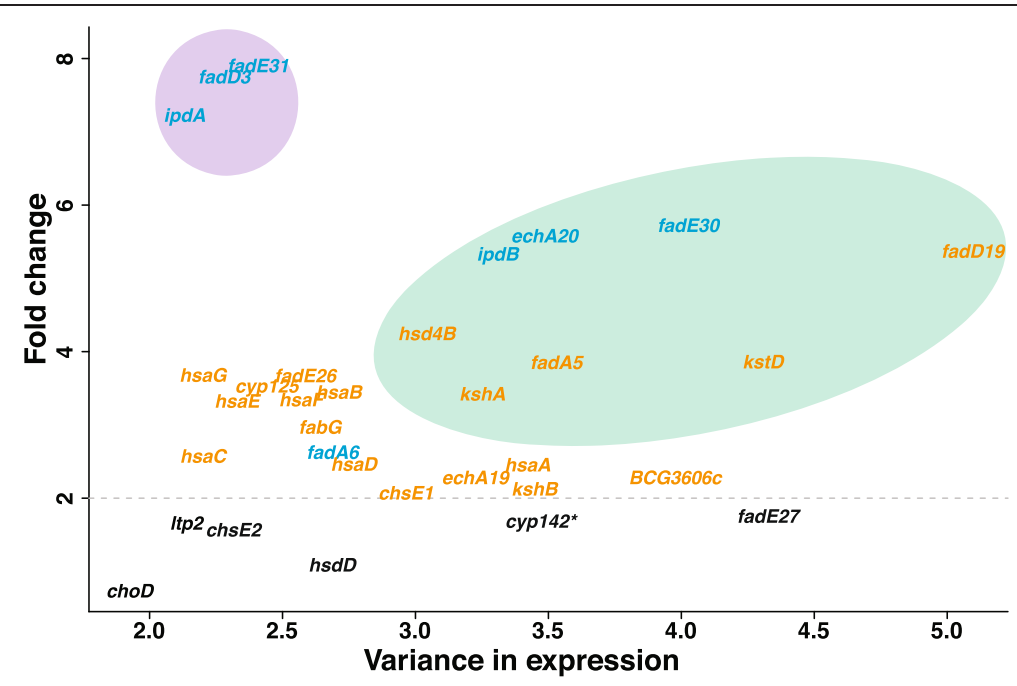

Figure 4 Variance in expression levels (compendium) compared to the fold changes upon infection. Genes in blue are KstR2-regulated whereas orange genes denote KstR-regulated genes. Genes in black show no significant change (FDR $>0.05$ ). Green/violet ellipses denote areas of high/low variability in the expression compendium. ${ }^{*}$ In M. tuberculosis H37Rv, cyp 142 has the same function as cyp125. In M. bovis BCG, cyp 142 encodes an inactive product.

resting or activated macrophages. The lack of induction of the sole asparaginase gene ansA (BCG1590c; Rv1538c), that can catalyze the conversion of asparagine to aspartate, suggests that, in addition to its reported asparagine transport capacity [37], mycobacterial AnsP2 imports aspartate from the phagosome during infection. Some of the genes that encode aspartate-utilizing enzymes are induced, such as panD and aspB (BCG3665c, BCG3629;
Rv3601c, Rv3565). In particular, AspB was predicted to transfer nitrogen from aspartate to glutamate, which serves as a central nitrogen carrier for alternative metabolic pathways [38], suggesting that $M$. bovis BCG utilizes host aspartate as nitrogen source during infection.

The repression of de novo $\mathrm{NAD}(\mathrm{P})$ synthesis genes nadA, nadB and nadC (BCG1632, BCG1633, BCG1634; Rv1594, Rv1595, Rv1596) and the absence of significant

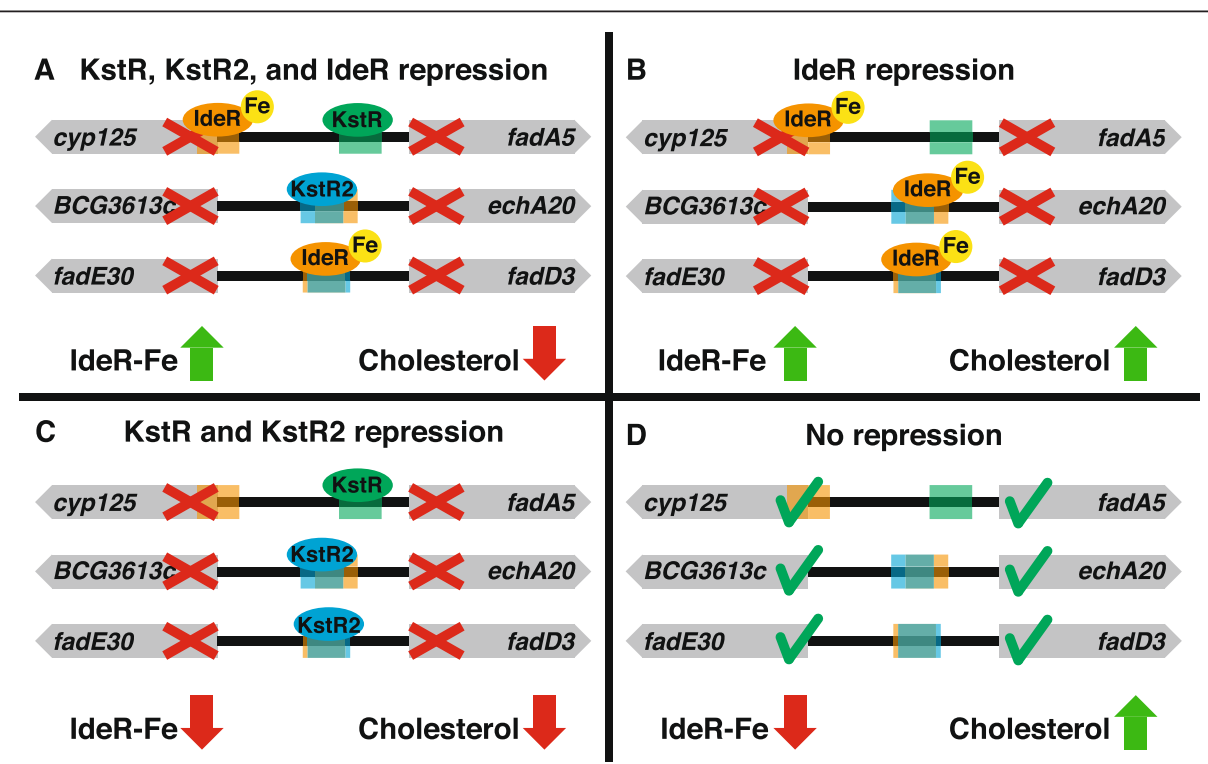

Figure $\mathbf{5}$ Regulation of the cholesterol degradation pathway by IdeR, KstR and KstR2. Sequences similar to the IdeR binding boxes appear in the upstream regions of genes in the cholesterol degradation pathway in close proximity to (and sometimes overlapping with) the KstR and KstR2 binding regions. (A, B and C) Under either normal iron availability or lack of cholesterol either IdeR or KstR/KstR2 represses the expression of genes in this pathway. (D) Only under low iron availability (relieving IdeR repression) and in presence of cholesterol (relieving KstR and KstR2 repression), can the genes in the cholesterol degradation pathway be expressed. 


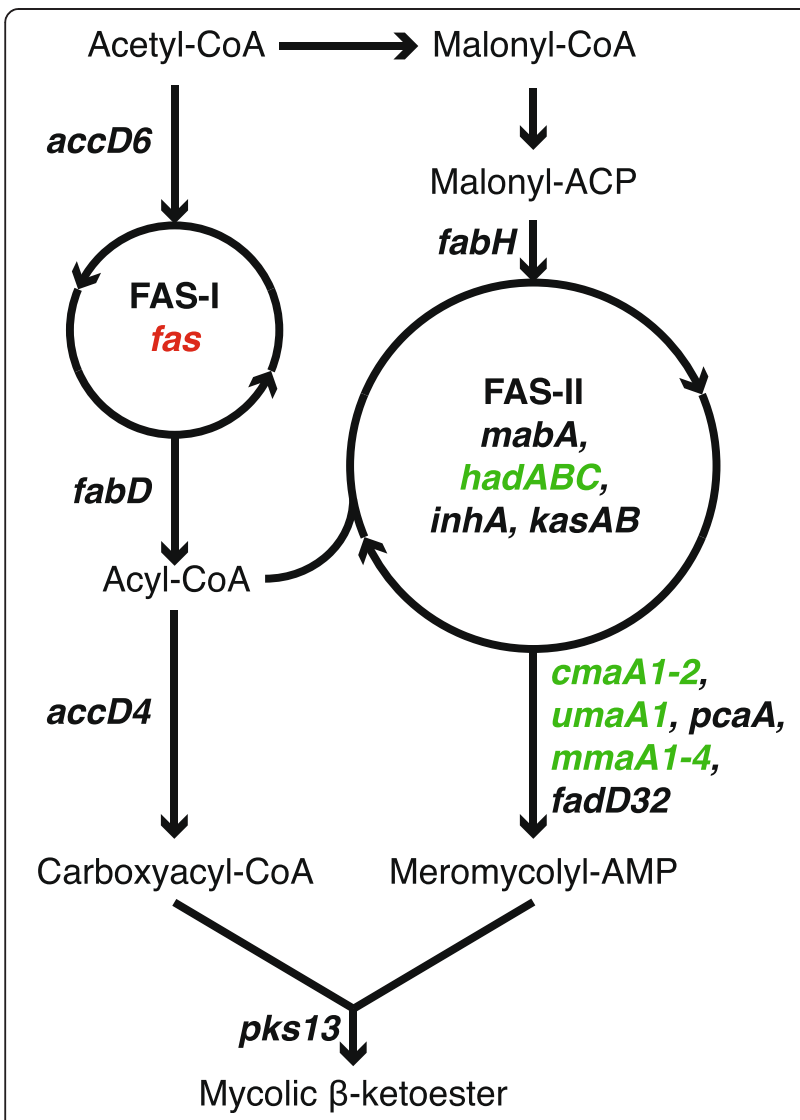

Figure 6 M. bovis BCG gene expression pattern of mycolic acid synthesis. Genes involved in fatty acid synthase II (FAS-II) and downstream of FAS-II are induced (green), while fatty acid synthase I (FAS-I) is repressed (red).

changes in expression of pncA, pncB, nadD and nadE (BCG2062c, BCG1392c, BCG2437c, BCG2457c; Rv2043c, Rv1330c, Rv2421c, Rv2438c) involved in NAD(P) synthesis and salvage [39] (Data set S1), indicates that bacterial $\mathrm{NAD}(\mathrm{P})$ may become limited during infection. The transcripts of enzymes catalyzing branching reactions towards threonine, methionine and lysine showed an unexpected pattern (Figure 3B): both dapA (BCG2769c; Rv2753c) and thrB (BCG1356; Rv1296), involved in initiation of threonine and lysine biosynthesis respectively were repressed, while metX (BCG3411; Rv3341), encoding an enzyme that initiates methionine biosynthesis, was induced. We conclude that host aspartate utilized by M. bovis BCG might largely be converted into methionine rather than threonine and lysine.

The induction of sodA (BCG3909; Rv3846) (Data set S1), encoding superoxide dismutase that destroys harmful radicals, confirms that $M$. bovis BCG counteracts reactive oxygen intermediates (ROI) produced by the host cell $[2,40]$. Interestingly, aspartate has the capacity to quench ROI by intramolecular oxidation of the sulphur atom [41]. Although experimental prove has yet to be provided, it is attractive to speculate that mycobacteria produce methionine during infection to support counteraction to ROI.

\section{$M$. bovis BCG iron scavenging; siderophore synthesis, secretion and import}

Mycobactins comprise an essential class of mycobacterial siderophore molecules to access iron of the host. These molecules are synthesized by an array of mycobactin enzymes, consisting of several proteins organized in a megasynthase [42]. The mycobactin megasynthase genes mbtB-F were induced upon infection and so were the majority of additional genes involved in mycobactin biosynthesis: $\mathrm{mbtG} / \mathrm{I} / \mathrm{J} / \mathrm{K} / \mathrm{N}$ (BCG2392c, BCG2400c, BCG2399, BCG1409c, BCG1408; Rv2378c, Rv2385, Rv2386c, Rv1347c, Rv1346) (Data set S1).

The type VII secretion system ESX-3 is essential for mycobactin-mediated iron acquisition and in vivo survival [43]. The ESX-3 secretion system is regulated by ZuR (BCG2373; Rv2359) [44] and consists of 11 genes [45] of which 7 were induced upon infection (Data set S1). The repression of $z u R$, resulted in the induction of ESX-3. A siderophore transport system of $M$. tuberculosis consisting of MmpL4 and MmpS4 (BCG0489c, BCG0490c; Rv0450c, Rv0451c) is required for infection of mice [46]. Both mmpL4 and mmpS4 and two other genes encoding an inner membrane transporter for mycobactin irtA/B [47] (BCG1410/1411; Rv1348/1349) were induced (Data set S1). Of the bacterioferritins $\mathrm{BfrA} / \mathrm{B}$ (iron storage proteins induced by IdeR), only bfrB (BCG3904; Rv3841) showed a significant decrease. A possible explanation could be the reduced availability of iron in the host, and thus less iron storage capacity is required.

\section{M. bovis BCG small RNAs}

Small RNAs have only recently been discovered in Mycobacteria $[48,49]$. Although their function is mostly unclear, they can be present in large quantities [50]. In our study, differential expression was observed for 19 small RNAs (FDR < 0.05). High transcript levels of the small RNAs MTS0997, MTS1338 and MTS2823 were reported in chronically $M$. tuberculosis-infected mouse lungs [50]. We observed a significant $(F D R<0.05)$ induction of MTS2823 as well, although the fold change is small $(\log F C=1.49)$ compared to other reports. The expression of MTS1338 was repressed in our study, and showed a small fold change $(\operatorname{logFC}=-2.03)$. DosR (BCG3156c; Rv3133c) induces the latter in M. tuberculosis upon hypoxia and infection [51]. The low expression (below $100 \mathrm{CPM}$ ) and lack of induction of dosR in our datasets, may explain why MTS1338 remained unchanged. We verified the RNA-seq data by qRT-PCR (Additional file 5). Whether the contrary expression of 
MTS1338 in M. bovis BCG and in M. tuberculosis during infection is critical for virulence remains to be defined.

Host immune response to $M$. bovis BCG is AIM2 dependent A pathway enrichment analysis using InnateDB [52] revealed that the THP-1 cells show distinct signs of infection (Table 2) since we identified numerous enriched pathways involved in immune response such as IFN- $\alpha / \beta$ signaling, IFN- $\gamma$ signaling and RIG-I/MDA5-mediated induction of IFN- $\alpha / \beta$ pathways. Interferons (IFNs) are synthesized by the host upon infection and trigger the activation of its immune system. IFNs can be divided in three classes: type I IFNs (IFN- $\alpha$, IFN- $\beta$, IFN- $\varepsilon$ and IFN- $\omega$ ), type II IFNs (IFN- $\gamma$ ) and type III IFNs [53].

Shah and colleagues [54] showed that virulent mycobacteria, such as $M$. tuberculosis inhibit IFN- $\beta$ production and signaling, resulting in the inhibition of the activation of AIM2 (interferon-inducible protein). AIM2 is part of the inflammasome that recognizes cytosolic bacterial and viral DNA, thereby contributing to the host's defense. In contrast to virulent mycobacteria, nonvirulent mycobacteria such as $M$. smegmatis, induce AIM2 [54]. M. bovis BCG seems to respond similarly to other nonvirulent mycobacteria, as the transcription of the gene encoding AIM2 is highly induced (Data set S1) as well as the IFN- $\alpha / \beta$ signaling pathway and the cytosolic DNA-sensing pathway (Table 2).

\section{Host genes involved in glycolysis and ketogenesis are induced upon mycobacterial infection}

Phagocytosis of pathogenic mycobacteria triggers the accumulation of lipid bodies in the host cell described as foamy phenotype [55]. Secretion of mycobacterial ESAT-6

Table 2 Induced THP-1 pathways upon M. bovis BCG infection

\begin{tabular}{|c|c|c|c|}
\hline Pathway name & $\begin{array}{l}\text { Number } \\
\text { of genes } \\
\text { annotated } \\
\text { in pathway }\end{array}$ & $\begin{array}{l}\text { Number } \\
\text { of induced } \\
\text { genes }\end{array}$ & P-value \\
\hline IFN- $\alpha / \beta$ signaling & 36 & 23 & $<1.0 \times 10^{-5}$ \\
\hline IFN- $\gamma$ signaling & 28 & 16 & $<1.0 \times 10^{-5}$ \\
\hline $\begin{array}{l}\text { RIG-I/MDA5-mediated induction } \\
\text { of IFN-a/ } \beta \text { pathways }\end{array}$ & 12 & 8 & $<1.0 \times 10^{-5}$ \\
\hline Cytosolic DNA-sensing pathway & 23 & 8 & 0.00018 \\
\hline Cholesterol biosynthesis & 14 & 6 & 0.0033 \\
\hline Hepatitis C & 71 & 14 & 0.00073 \\
\hline Staphylococcus aureus infection & 11 & 5 & 0.00078 \\
\hline Steroid biosynthesis & 11 & 5 & 0.00078 \\
\hline Iron uptake and transport & 7 & 4 & 0.00096 \\
\hline Toxoplasmosis & 60 & 12 & 0.00152 \\
\hline
\end{tabular}

The ten most significantly induced pathways are shown. is required to mediate this process by stimulating the uptake of glucose into the host cell, which might lead to increased glycolytic activity and elevated levels of acetylCoA, which in turn leads to the generation of D-3hydroxybutyrate via ketogenesis [55]. Although M. bovis BCG lost the ESX-1 locus, a major virulence determinant of pathogenic mycobacteria that encodes the effector proteins ESAT-6 and CFP-10 [13,56], we observe increased expression of several glycolytic enzymes including $\mathrm{HK} 3$, GPI, PFKP, FBPI, GAPDH, and PGAM1 (Additional file 2). Moreover, we found genes of the ketogenesis pathway induced: ACAT2, HMGCS1, BDH2 and HMGCR. The latter gene encodes HMG-CoA reductase, which catalyzes the conversion of hydroxy- $\beta$-methylglutaryl CoA and leads to the synthesis of cholesterol and other sterols. $\mathrm{BDH} 2$ encodes 3-hydroxybutyrate dehydrogenase, which catalyzes the reversible conversion of acetoacetate to D-3-hydroxybutyrate. Subsequent steps that lead to mycobacteria-induced formation of lipid bodies in the host cell involve the activation of the anti-lipolytic G protein-coupled receptor GPR109A, which triggers adenylyl cyclase. The resulting decrease in host cyclic AMP levels leads to a decrease in phosphorylation of stored host lipids by protein kinase A (PKA), rendering them less vulnerable to lipolysis by hormone sensitive lipase (HSL), thus promoting the formation of lipid bodies [55]. Consistent with the attenuated $M$. bovis BCG strain, we do not observe a change in the expression of GPR109A, adenylyl cyclase, PKA and HSL, indicating that this part of the pathway leading to the formation of lipid bodies is not active in the host or that this response is regulated posttranscriptionally and therefore remains invisible using a transcriptome approach.

Taken together, despite the absence of the ESX-1 locus in $M$. bovis BCG, the host response regarding the initial steps of lipid body formation is similar to that of $M$. $t u$ berculosis. Several pathogen factors including mycolic acids that were demonstrated to induce the foamy phenotype in macrophages [57], may therefore be required to reprogram the host for lipid build-up.

\section{Cholesterol is synthesized and iron losses are compensated by the host upon infection}

Four out of seven genes in the THP-1 iron uptake and transport pathway were induced. The enzyme encoded by the induced gene HMOX1 encodes heme oxygenase 1 , assigned to iron uptake and transport, catalyzes the rate-limiting step of heme degradation and is required to confer host resistance to mycobacterial infection in mice [58]. Among the other induced genes were FTH1 and FTL, encoding the heavy and light polypeptide of ferritin. This suggests that the THP-1 cells compensate for the loss of iron caused by $M$. bovis BCG, by taking up extra iron and degrading heme. 
The THP-1 cholesterol biosynthesis pathway was induced, as six genes of this pathway, including HMGCR encoding the rate-limiting enzyme for cholesterol biosynthesis, were upregulated. We conclude that infected macrophages synthesize cholesterol to compensate for loss of this molecule caused by mycobacterial catabolism.

\section{Discussion}

This study describes a deep sequencing approach towards the elucidation of mycobacterial and host cell gene expression profiles during intracellular infection. Initially we employed standard deep sequencing settings for eukaryotes to resolve the transcriptional profile of intracellular mycobacteria. Although this set-up allowed, to some extent, analyzing gene expression in $M$. bovis BCG, mycobacterial transcript coverage was insufficient. Increasing the sequencing depth was hindered by high sequencing costs, thereby preventing the method to become a broad application. Thus, we decided to enrich for mycobacteria during sample preparation of infected THP-1 cells. Indeed, the strategy applied greatly increased the coverage of the intracellular $M$. bovis BCG transcriptome. Although mycobacterial gene expression can be analyzed in non-enriched samples (215 genes, FDR $<0.05$ ), implementation of enrichment greatly expanded the number of reliably identified differentially expressed genes by $71 \%$ (367 genes, FDR < 0.05).

Moreover, the sequencing depth simulation (Figure 2B) revealed that enrichment allowed identification of differentially expressed genes that would have been missed otherwise. Repasy and colleagues showed that for an in vivo infection setting with mice and $M$. tuberculosis the MOI ranges from 1 to 5 [3]. Therefore, when studying an in vivo infection, the enrichment might not be sufficient to obtain a similar sequencing depth as obtained in our in vitro study, for which an MOI of 10 was used. To determine whether enrichment introduced any bias into the datasets, we analyzed non-enriched and enriched samples of two independent biological infection replicates. Although the non-enriched and enriched samples comprised different numbers of intracellular $M$. bovis BCG reads, the transcriptomes of respective samples of both infection experiments correlated well as indicated by high linearity and correlation coefficients (Additional file 1). Additionally, the correlation of intracellular M. bovis BCG expression between the biological replicates of both enriched datasets was comparable with the non-enriched datasets (0.93 and 0.94, respectively), verifying that the enrichment procedure was repeatable and robust, and did not introduce any bias to the intracellular mycobacterial transcriptome. For host expression we identified a lower correlation between the non-enriched and enriched samples ( 0.57 for IF1 and IF1ER, and 0.70 for IF2 and IF2ER) (Additional file 1). Hence, the datasets of non-enriched samples were favored for analysis of the host transcripts in order to preserve accuracy of the transcriptional landscape during infection.

Our method is dependent on differential susceptibility to lysis of host and microbial cells and not on a molecular sequence capture or depletion method as previously described [11]. This has the added advantage that small RNAs can be detected and analyzed for differential expression. Additionally, our method is independent of mRNA polyadenylation, as e.g. MICROBEnrich simultaneously captures and removes polyadenylated mRNAs along with rRNAs. We consider this a critical point because previous findings support the existence of polyadenylated tracts in mRNA of mycobacteria [59] and other bacteria [60,61].

It has been shown previously that mycolic acid liposomes are phagocytized by murine macrophages, changing the morphology of the macrophages to foam-like cells accumulating cholesterol [57]. Although the cholesterol degradation pathway is induced at 24 hours postinfection (Figure 3A and Additional file 3), the mce4 gene cluster and $h s d D$ and $c h o D$ were not induced. Nonetheless, these genes were expressed in both conditions and respective proteins could be already present before infection, ready for a situation when cholesterol becomes available. For $h s d D$ and $c h o D$, it is tempting to speculate that alternative genes with a similar function exist, since these genes have been found dispensable for cholesterol degradation in mycobacteria [21].

Gene regulation of mycobacterial cholesterol catabolism involves a complex interplay between KstR, KstR2, and IdeR. KstR and KstR2 are the prime regulators of the genes depicted in Figure 3A. A clear distinction between their targets becomes apparent with KstR regulating the degradation of rings $A$ and $B$ and KstR2 regulating the degradation of rings $C$ and $D$. In addition, genes in these regulons (fadA5, fadD3, fadE30, echA20) contain IdeRbinding sites in their upstream regions [33] and in some cases a profound overlap between these binding sites was observed. This implies that these genes can only be expressed upon removal of both types of repressors: IdeR under normal iron availability and KstR and KstR2 during cholesterol shortage (Figure 5). The upregulation of IdeRdependent iron uptake systems can be caused not only by low iron availability inside the macrophage but also as a response to the NO-induced damage caused to ironcontaining proteins [5]. HsaC, KshA, and the cytochromes Cyp125 and Cyp142 are iron-containing enzymes (and hence are susceptible to NO-induced damage) and lack of functionality of these enzymes leads to the accumulation of stable toxic catabolic intermediates, such as catechol derivatives and cholest-4-en-3-one [62-65]. The IdeR control of this pathway ensures that it is only expressed when the corresponding repair/replacement systems for iron- 
containing proteins are in place, therefore minimizing the risk of toxic intermediate accumulation.

It has been shown that aspartate functions as a major nitrogen reservoir in the host [36]. In line with this finding, we observed induction of aspartate utilizing enzymes (Figure 3B). Interestingly, we did not detect induction of the gene encoding the aspartate transporter ansP1, but induction of its homolog, ansP2. Earlier reports demonstrated that an ansP1-KO mutant fails to import aspartate in vitro, but shows wild-type behavior in either resting or activated macrophages, even though aspartate is a major nitrogen source in the host. Moreover, the absence of ansA induction suggests AnsP2 functions as an aspartate importer during infection in addition to its role as asparagine importer [37].

Rodríguez and colleagues cultivated $M$. tuberculosis $\mathrm{H} 37 \mathrm{Rv}$ on even long-chain fatty acids and analyzed the transcriptome by RNA sequencing, observing a dormancyrelated phenotype [66]. Although there are similarities between their in vitro model and our results, the induction of cholesterol catabolism, the methylcitrate cycle and aspartate metabolism are not captured using such a method, highlighting the differences between using an in vitro model based on even long chain fatty acids as opposed to studying intracellular infection directly.

\section{Conclusions}

Dual RNA-seq allowed elucidation of the complex interplay between M. bovis BCG and THP-1 macrophages. The comparison of non-enriched and enriched ribosomal RNA-depleted sequencing libraries of two biological replicates from identical infection cultures, showed high correlation of sequencing reads without technical bias. Taken together, microbe-enriched dual RNA-seq is a powerful technology that enables the assessment of the global transcriptome of "low-number" intracellular microbes and their host as demonstrated by the simultaneous induction of $M$. bovis BCG cholesterol degradation genes and host cholesterol synthesis genes.

\section{Methods}

\section{Bacterial strains and growth conditions}

M. bovis BCG SSI 1331 (American Type Culture Collection, \#35733) was grown in Middlebook 7H9 medium (Becton Dickinson) supplemented with $0.05 \%$ Tween 80, $0.2 \%$ glycerol, $10 \%$ albumin-dextrose-catalase supplement (Becton Dickinson) (7H9-ADC) or on Middlebrook 7H11 agar (Becton Dickinson) containing 0.2\% glycerol and 10\% oleic acid-albumin-dextrose-catalase enrichment (Becton Dickinson). Mycobacterial cultures were grown to the mid-log phase in $1 \mathrm{~L}$ roller bottles $\left(450 \mathrm{~cm}^{2}\right)$ at $37^{\circ} \mathrm{C}$ and $2 \mathrm{rpm}$. For CFU enumeration, serial dilutions were performed in phosphate-buffered saline containing 0.05\%
Tween 80 and plated on Middlebrook 7H11 agar. Plates were incubated at $37^{\circ} \mathrm{C}$ for $3-4$ weeks prior to counting.

\section{Infection of the human macrophage-like cell line THP-1}

The THP-1 cell line (American Type Culture Collection \#TIB-202) was maintained in Roswell Park Memorial Institute medium 1640 supplemented with $10 \%$ fetal calf serum, $2 \mathrm{mM}$ glutamine, $1 \mathrm{mM}$ sodium pyruvate and $0.05 \mathrm{mM} 2$-mercapto-ethanol in a humidified 5\% carbon dioxide atmosphere at $37^{\circ} \mathrm{C}$. Estimated $4 \times 10^{6}$ cells/well in a 6-well plate were differentiated for $24 \mathrm{~h}$ using culture medium containing $40 \mathrm{ng} / \mathrm{ml}$ phorbol 12 -myristate 13-acetate. Cells were then washed with fresh culture medium and incubated for 48 h. M. bovis BCG was pelleted (3,200 rpm, RT, $10 \mathrm{~min}$ ), washed twice with phosphate buffered saline and resuspended in THP-1 culture medium. THP-1 cells were infected with $M$. bovis BCG at a multiplicity of infection of 10 . The $M$. bovis BCG culture medium suspension was added to the differentiated cells, centrifuged for $3 \mathrm{~min}$ at $800 \mathrm{rpm}$ and subsequently incubated for $4 \mathrm{~h}$. The infection mix was removed, cells were washed twice with pre-warmed PBS and incubated with fresh culture medium for $20 \mathrm{~h}$ prior to RNA extraction.

\section{RNA isolation and mycobacterial RNA enrichment of infected cells}

Total RNA from mycobacterial cultures was prepared as previously described [67]. Extraction of total RNA from THP-1 cells was prepared with TRIzol reagent using glycogen as a carrier according to the suppliers' recommendation (Life Technologies). Total RNA from $M$. bovis BCG infected THP-1 cells was isolated by abrasive particles in a reciprocal shaker with TRIzol [67]. Enrichment of mycobacteria from infected THP-1 cells was carried out by differential lysis of host and mycobacterial cells by guanidine thiocyanate (GITC).

Infected cells were washed with PBS at RT. Cold $4 \mathrm{M}$ GITC was added to the monolayer and the cells were transferred to a $1.5-\mathrm{ml}$ screw cap tube. After centrifugation the pellet was resuspended in residual GITC and mixed with $1 \mathrm{ml}$ TRIzol containing $20 \mu \mathrm{g} / \mathrm{ml}$ linear acrylamide, followed by incubation for $5 \mathrm{~min}$ at RT. Bacteria were disrupted by bead beating (FastPrep Instrument; two cycles of 30s at maximum speed with cooling on ice between cycles). The sample was centrifuged for $1 \mathrm{~min}$ at $4^{\circ} \mathrm{C} / 13,000 \mathrm{rpm}$ and the supernatant was transferred to a 2-ml screw cap tube containing $200 \mu \mathrm{l}$ chloroform, mixed and incubated at RT for $5 \mathrm{~min}$. After centrifugation at $4^{\circ} \mathrm{C} / 13,000 \mathrm{rpm}$ for $10 \mathrm{~min}$, RNA was extracted from the aqueous phase using the Qiagen RNeasy mini kit including an on-column DNase digestion (Qiagen). Quality and quantity of total RNA were assessed 
using an Agilent 2100 Bioanalyzer (Agilent Technologies) and a NanoDrop 1000 spectrophotometer (Kisker).

\section{Calculation of spike-in concentration}

Total RNA of THP-1 cells and M. bovis BCG cultures was determined on the basis of cell counts and RNA isolation yield. As the intracellular copy number for a pathogen varies from species to species we assumed the most minimal infection rate of one mycobacterium per host cell. Therefore a cellular multiplicity of infection of 1:1 was used for mixing host and pathogen RNA, resulting in a ratio of 1000:1 total RNA, derived from the proportion of RNA abundance per cell between host and pathogen.

\section{RNA sequencing}

The RNA-seq libraries were prepared according to the TruSeq RNA Sample Preparation v2 Guide (Illumina) without fragmentation and without size selection. Using an Agilent Bioanalyzer high sensitivity DNA kit, we confirmed that the average final library size was already approximately $350 \mathrm{bp}$ without fragmentation; therefore the library insert fragmentation time at $94^{\circ} \mathrm{C}$ was set to $0 \mathrm{mi}-$ nutes, as no additional fragmentation step was required. Up to $98 \%$ of bacterial RNA consists of ribosomal RNA, which can prevent adequate coverage of a bacterial transcriptome, using RNA sequencing [68,69]. Therefore, the Gram-Positive Bacteria Ribo-Zero (Epicentre) rRNA Magnetic Removal Kit was used to remove bacterial rRNA from mycobacterial total RNA. For THP-1 total RNA the Ribo-Zero Magnetic Kit Human/Mouse/Rat was used, while depletion of rRNA from infections without enrichment as well as the spike-in experiment was done by a two-step procedure with both kits. The mycobacterial enriched total RNA preparations of infected cells were depleted with the Ribo-Zero Magnetic Gold Kit Epidemiology. All cDNA libraries were checked for quality using the DNA-1000 kit (Agilent) on a 2100 Bioanalyzer and quantified with the Qubit 2.0 Fluorometer (Life Technologies). Libraries of each consecutive experiment were pooled as 4-plex and on-board loaded with a Hi-Seq 1500 instrument. The sequencing reaction was carried out as Rapid Run using a TruSeq Rapid PE Cluster Kit and a TruSeq Rapid SBS Kit and $2 \times 51$ cycles including 7 cycles indexing in order to obtain 50-bp paired-end reads.

\section{Data analysis pipeline}

A total of eight datasets were created: Uninfected THP1 cells (dataset: THP), exponentially growing $M$. bovis BCG (dataset: EGB), a spike-in sample consisting of THP-1 RNA and $M$. bovis BCG RNA in a 1000:1 ratio (dataset: SPI), M. bovis BCG-infected THP-1 cells with a multiplicity of infection of $10 \mathrm{M}$. bovis BCG bacilli per
THP-1 host cell (dataset IF0), two additional independent biological replicates of $M$. bovis BCG-infected THP1 cells (datasets: IF1 and IF2), and two datasets prepared with the enrichment method using the two biological replicates of $M$. bovis BCG-infected THP-1 cells (datasets: IF1ER and IF2ER).

All 50-bp paired-end reads from datasets IF1/2ER, IF0/1/2, EGB were aligned, using megablast, against the complete genome sequence of $M$. bovis BCG Pasteur, obtained from the NCBI bacterial genome database (ftp. ncbi.hih.gov/genomes/Bacteria) and to the human transcriptome obtained from the Ensemble database (www. ensembl.org). If both 50-bp reads of a given pair could not be aligned to the human transcriptome, or the $M$. bovis BCG genome, they were discarded from further analysis. The 50-bp paired-end reads aligning to the $M$. bovis BCG genome were subsequently aligned to proteincoding genes, ribosomal genes, and small RNA (sRNA) genes. The protein-coding gene sequences and the ribosomal gene sequences were obtained from the NCBI database. The sRNA-coding gene sequences were obtained from the Bacterial Small Regulatory RNA Database [70] and supplemented with sRNA sequences from $M$. tuberculosis [51].

\section{Gene counting procedure}

A single count was assigned to a transcript if a complete pair of reads aligned to the $M$. bovis BCG genome. If only one read of a pair of reads aligned to a given gene, also a single count was assigned to this transcript, assuming that the other read could align to an intergenic region, or another gene, due to the existence of operons. If a pair of reads would align to two different genes, a count was assigned to both genes, resulting in a total of two counts per pair of reads. For THP-1 cells, all reads assigned to different splice variants of the same gene were counted (one count for each aligned read) and summed.

\section{Differential gene expression analysis}

The R package edgeR [71] was used for differential gene expression analysis of $M$. bovis BCG and THP-1 genes. Gene and protein functions have been extensively studied in M. tuberculosis and therefore the M. tuberculosis (strain H37Rv) orthologs of each M. bovis BCG gene were included. Low expression tags receiving less than 100 counts per million in two or more of the three datasets: EGB, IF1/2ER (or EGB, IF1/2), were excluded from differential expression analysis as described in the edge $R$ manual. Tags receiving more than 100 counts per million in the EGB dataset and less than 100 counts per million in either one or both replicates of IF1/2ER or IF $1 / 2$ were still used to account for large decreases in expression upon infection. 
EdgeR is a Bioconductor package designed to identify significant changes between two or more groups, given that at least one of the groups has replicated measurements [71], which is the case for our experimental setup (Figure 1). The edgeR algorithm models the read counts associated to a gene using a negative binomial probability distribution. The variance of this distribution takes into account both the stochasticity of the sequencing process and the variability associated to biological variation. The common Biological Coefficient of Variance $(\mathrm{BCV})$ measures the average dispersion in gene expression values associated to biological variability. We used EdgeR to compute the BCV of the corresponding samples prior to computing differential expression. For the BCG genes, we obtain a BCV of 0.16 from the IF1/2ER samples whereas for the THP-1 genes we obtained a BCV of 0.20 from the IF1/2 samples. We assumed that the $\mathrm{BCV}$ in datasets $\mathrm{EGB}$ and THP to be smaller than those in datasets IF1/2ER and IF1/2, due to the less stable and controlled conditions arising from infection as compared to standard culturing procedures. To compute differential gene expression for $M$. bovis BCG and THP-1 cells, we took a conservative approach and assigned the same BCV to datasets EGB and THP as those obtained from IF1/2ER and IF1/2, respectively. Differential expression was then computed for each gene using a pairwise exact testing procedure. The algorithm of Benjamini and Hochberg was used to control false discovery rates [72]. Protein-coding genes and sRNAcoding genes with an $\mathrm{FDR}<0.05$ were assumed to be differentially expressed.

For THP-1 cells, gene counts from dataset THP were compared to those of datasets IF1/2. Differential gene expression analysis was performed similar to $M$. bovis BCG. The low expression tags cutoff of 100 counts per million was normalized for the number of genes in the THP-1 genome. Afterwards, we used InnateDB and pathway enrichment analysis to identify induced host pathways [52].

\section{Sequencing depth simulation}

Subsets of 50-bp sequencing reads, where both pairs aligned to the complete genome of $M$. bovis BCG, were taken randomly from dataset EGB and IF1ER. These subsets were of the same size $(3.35 \mathrm{M})$ as the total number of sequencing reads from dataset IF2ER aligning to the $M$. bovis BCG genome in pairs (Figure 2A). From these two subsets, and from the paired-end sequencing reads of IF2ER aligning to the $M$. bovis BCG genome, random sets of reads were chosen of different sizes. For each different size, five random sets were generated. Differential gene expression for $M$. bovis BCG was determined using the method described above (data analysis pipeline) for every set. The mean and standard deviation of the number of differentially expressed genes was calculated (Figure 2B).

\section{Compendium of expression data}

A compendium containing 565 two-color microarrays for M. tuberculosis (strain H37Rv) was obtained from literature [73] and most of these (454) captured the effect of 75 drugs targeting metabolic pathways [74,75] whereas 111 captured stress-induced dormancy in the wild-type and in DosR activation genes in $\mathrm{KO}$ mutants [76,77].

\section{Quantitative real time PCR}

To verify the expression of several genes and small RNA's, their expression was determined using qRT-PCR. For 13 genes, we designed primers (Additional file 4) and quantified their relative expression to the reference gene: $r p o B$ (BCG0716; Rv0667). This gene encodes the RNA polymerase $\beta$-subunit and is thought to be a housekeeping gene, which was used as such in previous qRT-PCR studies [78-80]. Fold changes in gene expression (Additional file 5) were calculated using the $\Delta \Delta \mathrm{Ct}$, as described previously [81].

\section{Availability of supporting data}

Raw sequence read data supporting the results of this article are available in the EMBL-EBI European Nucleotide Archive under the Accession No. PRJEB6552, http://www. ebi.ac.uk/ena/data/view/PRJEB6552.

\section{Additional files}

Additional file 1: Comparison between non-enriched and enriched datasets. The normalized counts of each gene for both, M. bovis BCG ( $A$ and $B$ ) and THP-1 cells ( $C$ and D), of the non-enriched datasets were plotted versus the enriched datasets. The Pearson's correlation coefficients are denoted by an $r$ in the lower right corner of each plot. The correlations for $M$. bovis BCG genes between non-enriched and enriched datasets, are higher than those of the THP-1 genes.

Additional file 2: Differential expression of $M$. bovis BCG genes. Excel file with all expressed $M$. bovis BCG protein-coding genes, $M$. bovis BCG small RNA genes, THP-1 genes, induced THP-1 pathways and repressed THP-1 pathways.

Additional file 3: Cholesterol degradation pathway. Genes in green are induced upon infection (FDR $<0.05$ ), genes in black show no differential expression. Dashed arrows represent multiple reactions. The degradation of the ring $C$ and $D$ side chain is based on homologous genes from Rhodococcus equi. AD: 4-androstenedione, ADD: 1,4-androstenedione, 9OHADD: 9-hydroxy-1,4, androstene-3-17-dione, 3-HSA: 3-hydroxy-9, 10-seconandrost-1,3,5(10)-triene-9,17-dione 3,4-DHSA: 3,4-dihydroxy-9, 10-seconandrost-1,3,5(10)-triene-9,17-dione 4,9 DSHA: 4,5-9,10-diseco-3hydroxy-5,9,17-trioxoandrosta-1(10),2-diene-4-oic acid, HDD: 2-hydroxyhexa-2,4-dienoic acid, HIP: 9,17-dioxo-1,2,3,4,10,19-hexanorandrostan-5-oic acid, 5OH-HIP: 5-hydroxy-methylhexahydro-1-indanone propionate. *In M. tuberculosis H37Rv, cyp 142 has the same function as cyp 125. In M. bovis BCG, cyp 142 contains a single nucleotide deletion resulting in a premature stop codon and an inactive gene product. This gene (cyp142a) is two-fold induced upon infection.

Additional file 4: Primers used for qPCR. Word document containing 14 primer pairs used for QPCR of selected M. bovis BCG genes. 
Additional file 5: Differential expression of selected $M$. bovis BCG genes using qPCR. Excel file with Ct numbers for a variety of selected genes and sRNAs of $M$. bovis BCG. Fold changes in expression were calculated for these genes and sRNAs.

Additional file 6: Central carbon metabolism. Genes in green are induced, and genes in red are repressed upon infection (FDR $<0.05$ ), genes in black show no differential expression. The genes in the methylcitrate cycle are induced upon infection. ${ }^{*}$ In M. tuberculosis H37Rv, $i c / 2$ is fragmented into $a c e A a$ and $a c e A b$ and non-functional.

\section{Competing interests}

The authors declare that they have no competing interests.

\section{Authors' contributions}

RAR drafted the manuscript and performed the data analysis. MSD participated in drafting the manuscript, and helped with the data analysis, HJM, AD, GMD and GKS developed the enrichment method. HJM carried out the enrichment, RNA extraction and sequencing, and participated in drafting the manuscript. MG carried out the growth and infection experiments and participated in drafting the manuscript. SHEK, MG, VAPM and HJM conceived the study. PJS participated in conceiving the study and helped with the data analysis. All authors critically read, revised and approved the manuscript.

\section{Acknowledgements}

The authors thank Doris Lazar, Sabine Jörg, Ina Wagner and Karin Hahnke for technical support and Mary Louise Grossman for editorial assistance. The methodology to enrich bacterial transcripts from infected macrophages has been developed with support from the NIH/NIAID, contract \# HHSN272200800059C "A Systems Biology Approach to Infectious Diseases Research". This work has been supported by Framework Program 7 of the European Research Council, SysteMTb Collaborative Project (HEALTH-2009-2.1.1-1-241587) and by the Netherlands Consortium for Systems Biology (NCSB) which is part of the Netherlands Genomics Initiative / Netherlands Organisation for Scientific Research.

\section{Author details \\ 'Laboratory of Systems and Synthetic Biology, Wageningen University and Research Centre, Dreijenplein 10, 6703, HB, Wageningen, the Netherlands. ${ }^{2}$ Core Facility Microarray/Genomics, Max Planck Institute for Infection Biology, Charitéplatz 1, 10117 Berlin, Germany. ${ }^{3}$ Department of Microbiology and Immunology, Stanford University School of Medicine, 300 Pasteur Drive, Stanford, CA 94305-5124, USA. ${ }^{4}$ Department of Immunology, Max Planck Institute for Infection Biology, Charitéplatz 1, 10117 Berlin, Germany. ${ }^{5}$ LifeGlimmer GmbH, Markelstrasse 38, 12163 Berlin, Germany. ${ }^{6}$ Present address: Department of Microbiology, Yong Loo Lin School of Medicine, National University of Singapore, 5 Science Drive 2, Singapore 117545, Singapore.}

\section{Received: 6 June 2014 Accepted: 22 December 2014} Published online: 05 February 2015

\section{References}

1. World Health Organization. Global Tuberculosis Report 2013. Geneva: World Health Organization; 2013.

2. Gengenbacher M, Kaufmann SHE. Mycobacterium tuberculosis: success through dormancy. FEMS Microbiol Rev. 2012;36:514-32.

3. Repasy T, Lee J, Marino S, Martinez N, Kirschner DE, Hendricks G, et al. Intracellular bacillary burden reflects a burst size for Mycobacterium tuberculosis in vivo. PLoS Pathog. 2013;9:e1003190.

4. Westermann AJ, Gorski SA, Vogel J. Dual RNA-seq of pathogen and host. Nat Rev Micro. 2012;10:618-30

5. Schnappinger D, Ehrt S, Voskuil MI, Liu Y, Mangan JA, Monahan IM, et al. Transcriptional Adaptation of Mycobacterium tuberculosis within Macrophages: Insights into the Phagosomal Environment. J Exp Med. 2003:198:693-704

6. Rachman H, Strong M, Schaible U, Schuchhardt J, Hagens K, Mollenkopf H, et al. Mycobacterium tuberculosis gene expression profiling within the context of protein networks. Microbes Infect. 2006;8:747-57.
7. Fontan $P$, Aris $V$, Ghanny $S$, Soteropoulos $P$, Smith I. Global transcriptional profile of Mycobacterium tuberculosis during THP-1 human macrophage infection. Infect Immun. 2008;76:717-25.

8. Cappelli G, Volpe E, Grassi M, Liseo B, Colizzi V, Mariani F. Profiling of Mycobacterium tuberculosis gene expression during human macrophage infection: upregulation of the alternative sigma factor $\mathrm{G}$, a group of transcriptional regulators, and proteins with unknown function. Res Microbiol. 2006;157:445-55.

9. Dubnau E, Chan J, Mohan VP, Smith I. Responses of Mycobacterium tuberculosis to growth in the mouse lung. Infect Immun. 2005:73:3754-7.

10. Kuczynski J, Costello EK, Nemergut DR, Zaneveld J, Lauber CL, Knights D, et al. Direct sequencing of the human microbiome readily reveals community differences. Genome Biol. 2010;11:210.

11. Lamont EA, Xu WW, Sreevatsan S. Host-Mycobacterium avium subsp. paratuberculosis interactome reveals a novel iron assimilation mechanism linked to nitric oxide stress during early infection. BMC Genomics. 2013;14:694

12. Garnier T, Eiglmeier K, Camus JC, Medina N, Mansoor H, Pryor M, et al. The complete genome sequence of Mycobacterium bovis. Proc Natl Acad Sci U S A. 2003;100:7877-82.

13. Brosch R, Gordon SV, Garnier T, Eiglmeier K, Frigui W, Valenti P, et al. Genome plasticity of BCG and impact on vaccine efficacy. Proc Natl Acad Sci U S A. 2007;104:5596-601.

14. Cole ST, Brosch R, Parkhill J, Garnier T, Churcher C, Harris D, et al. Deciphering the biology of Mycobacterium tuberculosis from the complete genome sequence. Nature. 1998;393:537-44.

15. Mangan JA, Monahan IM, Butcher PD. Gene expression during hostpathogen interactions: Approaches to bacterial mRNA extraction and labelling for microarray analysis. In: Wren BW, Dorrell N, editors. Methods in Microbiology vol 33: Functional Microbial Genomics. London: Academic; 2002. p. 137-51.

16. Talaat AM, Lyons R, Howard ST, Johnston SA. The temporal expression profile of Mycobacterium tuberculosis infection in mice. Proc Natl Acad Sci U S A. 2004;101:4602-7.

17. Oliver HF, Orsi RH, Ponnala L, Keich U, Wang W, Sun Q, et al. Deep RNA sequencing of $L$. monocytogenes reveals overlapping and extensive stationary phase and sigma B-dependent transcriptomes, including multiple highly transcribed noncoding RNAs. BMC Genomics. 2009;10:641.

18. Perkins TT, Kingsley RA, Fookes MC, Gardner PP, James KD, Yu L, et al. A strand-specific RNA-Seq analysis of the transcriptome of the typhoid bacillus Salmonella typhi. PLoS Genet. 2009;5:e1000569.

19. Yoder-Himes DR, Chain PS, Zhu Y, Wurtzel O, Rubin EM, Tiedje JM, et al. Mapping the Burkholderia cenocepacia niche response via high-throughput sequencing. Proc Natl Acad Sci U S A. 2009;106:3976-81.

20. Ouellet $\mathrm{H}$, Johnston JB, de Montellano PR. Cholesterol catabolism as a therapeutic target in Mycobacterium tuberculosis. Trends Microbiol. 2011;19:530-9.

21. Brzostek A, Rumijowska-Galewicz A, Dziadek B, Wojcik EA, Dziadek J. ChoD and $\mathrm{HsdD}$ can be dispensable for cholesterol degradation in mycobacteria. J Steroid Biochem Mol Biol. 2013;134:1-7.

22. Casabon I, Crowe AM, Liu J, Eltis LD. FadD3 is an acyl-CoA synthetase that initiates catabolism of cholesterol rings $C$ and $D$ in actinobacteria. Mol Microbiol. 2013;87:269-83.

23. Thomas ST, Sampson NS. Mycobacterium tuberculosis utilizes a unique heterotetrameric structure for dehydrogenation of the cholesterol side chain. Biochemistry. 2013;52:2895-904.

24. Voskuil MI. Mycobacterium tuberculosis cholesterol catabolism requires a new class of acyl coenzyme A dehydrogenase. J Bacteriol. 2013;195:4319-21.

25. Wipperman MF, Yang M, Thomas ST, Sampson NS. Shrinking the FadE Proteome of Mycobacterium tuberculosis: Insights into Cholesterol Metabolism through Identification of an a2 32 Heterotetrameric Acyl Coenzyme A Dehydrogenase Family. J Bacteriol. 2013;195:4331-41.

26. van der Geize R, Grommen AW, Hessels Gl, Jacobs AA, Dijkhuizen L. The steroid catabolic pathway of the intracellular pathogen Rhodococcus equi is important for pathogenesis and a target for vaccine development. PLoS Pathog. 2011;7:e1002181.

27. Driscoll MD, McLean KJ, Levy C, Mast N, Pikuleva IA, Lafite P, et al. Structural and biochemical characterization of Mycobacterium tuberculosis CYP142: evidence for multiple cholesterol 27-hydroxylase activities in a human pathogen. J Biol Chem. 2010;285:38270-82.

28. Wipperman MF, Sampson NS, Thomas ST. Pathogen roid rage: Cholesterol utilization by Mycobacterium tuberculosis. Crit Rev Biochem Mol Biol. 2014;49:269-93. 
29. Rienksma RA, Suarez-Diez M, Spina L, Schaap PJ, Martins dos Santos VAP. Systems-level modeling of mycobacterial metabolism for the identification of new (multi-)drug targets. Semin Immunol. 2014;26:610-22.

30. Pandey AK, Sassetti CM. Mycobacterial persistence requires the utilization of host cholesterol. Proc Natl Acad Sci. 2008;105:4376-80.

31. de Souza GA, Leversen NA, Malen H, Wiker HG. Bacterial proteins with cleaved or uncleaved signal peptides of the general secretory pathway. J Proteome. 2011;75:502-10.

32. Kendall SL, Burgess P, Balhana R, Withers M, Ten Bokum A, Lott JS, et al. Cholesterol utilization in mycobacteria is controlled by two TetR-type transcriptional regulators: $k s t R$ and kstR2. Microbiology. 2010;156:1362-71.

33. Yellaboina S, Ranjan S, Vindal V, Ranjan A. Comparative analysis of iron regulated genes in mycobacteria. FEBS Lett. 2006;580:2567-76.

34. Griffin JE, Pandey AK, Gilmore SA, Mizrahi V, McKinney JD, Bertozzi CR, et al. Cholesterol catabolism by Mycobacterium tuberculosis requires transcriptional and metabolic adaptations. Chem Biol. 2012;19:218-27.

35. Marrakchi H, Lanéelle M-A, Daffé M. Mycolic acids: structures, biosynthesis, and beyond. Chem Biol. 2014;21:67-85.

36. Gouzy A, Larrouy-Maumus G, Wu T-D, Peixoto A, Levillain F, Lugo-Villarino G et al. Mycobacterium tuberculosis nitrogen assimilation and host colonization require aspartate. Nat Chem Biol. 2013;9:674-6.

37. Gouzy A, Larrouy-Maumus G, Bottai D, Levillain F, Dumas A, Wallach JB, et al. Mycobacterium tuberculosis exploits asparagine to assimilate nitrogen and resist acid stress during infection. PLoS Pathog. 2014;10:e1003928.

38. Gouzy A, Poquet $Y$, Neyrolles $O$. A central role for aspartate in Mycobacterium tuberculosis physiology and virulence. Front Cell Infect Microbiol. 2013:3:68

39. Vilcheze C, Weinrick B, Wong KW, Chen B, Jacobs Jr WR. NAD+ auxotrophy is bacteriocidal for the tubercle bacilli. Mol Microbiol. 2010;76:365-77.

40. Lee WL, Gold B, Darby C, Brot N, Jiang X, de Carvalho LP, et al. Mycobacterium tuberculosis expresses methionine sulphoxide reductases $A$ and $B$ that protect from killing by nitrite and hypochlorite. Mol Microbiol. 2009; 71:583-93.

41. Weissbach H, Etienne F, Hoshi T, Heinemann SH, Lowther WT, Matthews B, et al. Peptide Methionine Sulfoxide Reductase: Structure, Mechanism of Action, and Biological Function. Arch Biochem Biophys. 2002;397:172-8.

42. McMahon MD, Rush JS, Thomas MG. Analyses of MbtB, MbtE, and MbtF suggest revisions to the mycobactin biosynthesis pathway in Mycobacterium tuberculosis. J Bacteriol. 2012;194:2809-18.

43. Siegrist MS, Unnikrishnan M, McConnell MJ, Borowsky M, Cheng TY, Siddiqi $\mathrm{N}$, et al. Mycobacterial Esx-3 is required for mycobactin-mediated iron acquisition. Proc Natl Acad Sci U S A. 2009;106:18792-7.

44. Maciag A, Dainese E, Rodriguez GM, Milano A, Provvedi R, Pasca MR, et al. Global analysis of the Mycobacterium tuberculosis Zur (FurB) regulon. J Bacteriol. 2007;189:730-40.

45. Bitter W, Houben EN, Bottai D, Brodin P, Brown EJ, Cox JS, et al. Systematic genetic nomenclature for type VII secretion systems. PLoS Pathog. 2009;5:e1000507.

46. Wells RM, Jones CM, Xi Z, Speer A, Danilchanka O, Doornbos KS, et al. Discovery of a siderophore export system essential for virulence of Mycobacterium tuberculosis. PLoS Pathog. 2013;9:e1003120.

47. Ryndak MB, Wang S, Smith I, Rodriguez GM. The Mycobacterium tuberculosis high-affinity iron importer, IrtA, contains an FAD-binding domain. J Bacteriol. 2010;192:861-9.

48. DiChiara JM, Contreras-Martinez LM, Livny J, Smith D, McDonough KA, Belfort M. Multiple small RNAs identified in Mycobacterium bovis BCG are also expressed in Mycobacterium tuberculosis and Mycobacterium smegmatis. Nucleic Acids Res. 2010;38:4067-78

49. Tsai C-H, Baranowski C, Livny J, McDonough KA, Wade JT, Contreras LM. Identification of Novel sRNAs in Mycobacterial Species. PLoS One. 2013:8:e79411.

50. Arnvig KB, Comas I, Thomson NR, Houghton J, Boshoff HI, Croucher NJ, et al. Sequence-based analysis uncovers an abundance of non-coding RNA in the total transcriptome of Mycobacterium tuberculosis. PLoS Pathog. 2011;7:e1002342

51. Arnvig K, Young D. Non-coding RNA and its potential role in Mycobacterium tuberculosis pathogenesis. RNA Biol. 2012;9:427-36.

52. Lynn DJ, Winsor GL, Chan C, Richard N, Laird MR, Barsky A, et al. InnateDB: facilitating systems-level analyses of the mammalian innate immune response. Mol Syst Biol. 2008:4:218.

53. de Weerd NA, Samarajiwa SA, Hertzog PJ. Type I interferon receptors: biochemistry and biological functions. J Biol Chem. 2007;282:20053-7.
54. Shah S, Bohsali A, Ahlbrand SE, Srinivasan L, Rathinam VA, Vogel SN, et al. Cutting edge: Mycobacterium tuberculosis but not nonvirulent mycobacteria inhibits IFN- $\beta$ and AIM2 inflammasome-dependent IL-1 $\beta$ production via its ESX-1 secretion system. J Immunol. 2013;191:3514-8.

55. Singh V, Jamwal S, Jain R, Verma P, Gokhale R, Rao Kanury VS. Mycobacterium tuberculosis-Driven Targeted Recalibration of Macrophage Lipid Homeostasis Promotes the Foamy Phenotype. Cell Host Microbe. 2012;12:669-81.

56. Bottai D, Di Luca M, Majlessi L, Frigui W, Simeone R, Sayes F, et al. Disruption of the ESX-5 system of Mycobacterium tuberculosis causes loss of PPE protein secretion, reduction of cell wall integrity and strong attenuation. Mol Microbiol. 2012:83:1195-209.

57. Korf J, Stoltz A, Verschoor J, De Baetselier P, Grooten J. The Mycobacterium tuberculosis cell wall component mycolic acid elicits pathogen-associated host innate immune responses. Eur J Immunol. 2005:35:890-900.

58. Silva-Gomes S, Appelberg R, Larsen R, Soares MP, Gomes MS. Heme catabolism by heme oxygenase-1 confers host resistance to mycobacterium infection. Infect Immun. 2013;81:2536-45.

59. Rindi L, Lari N, Gil MG, Garzelli C. Oligo(dT)-primed synthesis of CDNA by reverse transcriptase in mycobacteria. Biochem Biophys Res Commun. 1998:248:216-8.

60. Sarkar N. Polyadenylation of mRNA in prokaryotes. Annu Rev Biochem. 1997:66:173-97.

61. Mohanty BK, Kushner SR. Bacterial/archaeal/organellar polyadenylation. Wiley Interdiscip Rev RNA. 2011;2:256-76.

62. Chang JC, Miner MD, Pandey AK, Gill WP, Harik NS, Sassetti CM, et al. igr Genes and Mycobacterium tuberculosis cholesterol metabolism. J Bacteriol. 2009;191:5232-9.

63. Yam KC, D'Angelo I, Kalscheuer R, Zhu H, Wang JX, Snieckus V, et al. Studies of a ring-cleaving dioxygenase illuminate the role of cholesterol metabolism in the pathogenesis of Mycobacterium tuberculosis. PLoS Pathog. 2009;5:e1000344.

64. Ouellet H, Guan S, Johnston JB, Chow ED, Kells PM, Burlingame AL, et al. Mycobacterium tuberculosis CYP125A1, a steroid C27 monooxygenase that detoxifies intracellularly generated cholest-4-en-3-one. Mol Microbiol. 2010;77:730-42.

65. Petrusma M, Dijkhuizen $L$, van der Geize R. Structural features in the KshA terminal oxygenase protein that determine substrate preference of 3-ketosteroid 9a-hydroxylase enzymes. J Bacteriol. 2012;194:115-21.

66. Rodríguez JG, Hernández AC, Helguera-Repetto C, Aguilar Ayala D, Guadarrama-Medina R, Anzóla JM, et al. Global adaptation to a lipid environment triggers the dormancy-related phenotype of Mycobacterium tuberculosis. mBio. 2014;5:e01125-14.

67. Dietrich G, Schaible UE, Diehl K-D, Mollenkopf H-J, Wiek S, Hess J, et al, Isolation of RNA from mycobacteria grown under in vitro and in vivo conditions. FEMS Microbiol Lett. 2000;186:177-80.

68. Peano C, Pietrelli A, Consolandi C, Rossi E, Petiti L, Tagliabue L, et al. An efficient rRNA removal method for RNA sequencing in GC-rich bacteria. Microbial Informatics Experimentation. 2013;3:1

69. Giannoukos G, Ciulla D, Huang K, Haas B, Izard J, Levin J, et al. Efficient and robust RNA-seq process for cultured bacteria and complex community transcriptomes. Genome Biol. 2012;13:r23.

70. Li L, Huang D, Cheung MK, Nong W, Huang Q, Kwan HS. BSRD: a repository for bacterial small regulatory RNA. Nucleic Acids Res. 2013;41:D233-8.

71. Robinson MD, McCarthy DJ, Smyth GK. edgeR: a Bioconductor package for differential expression analysis of digital gene expression data. Bioinformatics. 2010;26:139-40.

72. Benjamini $Y$, Hochberg $Y$. Controlling the false discovery rate: a practical and powerful approach to multiple testing. J R Stat Soc Ser B Methodol. 1995:57:289-300

73. van Dam JCJ, Schaap PJ, Martins dos Santos VAP, Suarez-Diez M. Integration of heterogeneous molecular networks to unravel gene-regulation in Mycobacterium tuberculosis. BMC Syst Biol. 2014;8:111.

74. Boshoff HI, Myers TG, Copp BR, McNeil MR, Wilson MA, Barry 3rd CE. The transcriptional responses of Mycobacterium tuberculosis to inhibitors of metabolism: novel insights into drug mechanisms of action. J Biol Chem. 2004;279:40174-84

75. Karakousis PC, Williams EP, Bishai WR. Altered expression of isoniazidregulated genes in drug-treated dormant Mycobacterium tuberculosis. J Antimicrob Chemother. 2008;61:323-31.

76. Deb C, Lee CM, Dubey VS, Daniel J, Abomoelak B, Sirakova TD, et al. A novel in vitro multiple-stress dormancy model for Mycobacterium tuberculosis 
generates a lipid-loaded, drug-tolerant, dormant pathogen. PLoS One. 2009:4:e6077.

77. Honaker RW, Leistikow RL, Bartek IL, Voskuil MI. Unique roles of DosT and DosS in DosR regulon induction and Mycobacterium tuberculosis dormancy. Infect Immun. 2009;77:3258-63.

78. Adékambi T, Drancourt M, Raoult D. The rpoB gene as a tool for clinical microbiologists. Trends Microbiol. 2009;17:37-45.

79. Badejo AC, Badejo AO, Shin KH, Chai YG. A gene expression study of the activities of aromatic ring-cleavage dioxygenases in Mycobacterium gilvum PYR-GCK to changes in salinity and pH during pyrene degradation. PLoS One. 2013;8:e58066.

80. Lim SY, Kim BJ, Lee MK, Kim K. Development of a real-time PCR-based method for rapid differential identification of Mycobacterium species. Lett Appl Microbiol. 2008;46:101-6.

81. Yuan J, Reed A, Chen F, Stewart CN. Statistical analysis of real-time PCR data BMC bioinformatics. 2006;7:85.

\section{Submit your next manuscript to BioMed Central and take full advantage of:}

- Convenient online submission

- Thorough peer review

- No space constraints or color figure charges

- Immediate publication on acceptance

- Inclusion in PubMed, CAS, Scopus and Google Scholar

- Research which is freely available for redistribution 